\title{
MYOTONIA, A NEW INHERITED MUSCLE DISEASE IN MICE ${ }^{1}$
}

\author{
ALLEN H. HELLER, ${ }^{*}, 2$ EVA M. EICHER $\ddagger$ MARK HALLETT, $\S$ AND RICHARD L. SIDMAN* \\ ${ }^{*}$ Departments of Neurology-Neuropathology and Neuroscience, Children's Hospital Medical Center, §Section of Neurology, \\ Department of Medicine, Brigham and Women's Hospital, Harvard Medical School, Boston, Massachusetts 02115 and \\ $\ddagger$ The Jackson Laboratory, Bar Harbor, Maine 04609
}

Received December 1, 1981; Revised February 4, 1982; Accepted February 8, 1982

\begin{abstract}
Electromyographic (EMG) and histological studies were carried out with the new mouse mutant myotonia (mto, autosomal recessive). Affected animals exhibited behavioral myotonia which was apparent at 2 weeks of age. EMG studies revealed myotonic discharges (prolonged repetitive discharges with recurrent variation in amplitude and frequency) in all skeletal muscles tested. These discharges were not affected by peripheral nerve section or by pancuronium bromide, a neuromuscular synapse inhibitor. Qualitative histological study revealed no evidence of skeletal muscle fiber necrosis, grouped atrophy, or inflammation compared to sex-, age-, and weight-matched controls. Degeneration of myelinated axons in the ventrolateral funiculus of the spinal cord occurred only in older animals and does not explain the functional abnormality of muscle. Both the clinical and electrophysiological features of classical myotonia can be inherited as a single locus autosomal recessive disease in mice.
\end{abstract}

Myotonia is a feature of several human diseases and represents the primary manifestation of human myotonia congenita (Munsat and Scheife, 1979) and a congenital disease of goats (Brown and Harvey, 1939). Although myotonia-like activity has been described in dystrophia muscularis (both the $d y$ and $d y^{2 J}$ alleles) mutant mice (Gilbert et al., 1973; Eberstein et al., 1975), classical myotonia has not been well documented in any species other than goat and man.

Several years ago, a new mutation arose spontaneously at The Jackson Laboratory, Bar Harbor in the SWR/J inbred strain. Propagation of the mutant demonstrated an autosomal recessive mode of inheritance with full penetrance. The salient neuromuscular expression was an abnormal sustained posture of the hindlimbs provoked

\footnotetext{
${ }^{1}$ We wish to thank Ms. Vicky Cousins for bringing the mutation to our attention, Dr. Tessa Hedley-Whyte for valuable suggestions, Dr. Earl Henry for assistance with the surgical dissections, Mr. Richard Altschuler for preparing the histological specimens, and Mr. Mark Grove for technical assistance. This work was supported in part by Research Grants NS 11237 and RR01183 and Mental Retardation Center Grant HD06276 from the National Institutes of Health. Dr. Heller is the recipient of National Institute of Neurological and Communicative Disorders and Stroke Teacher-Investigator Development Award K07-NS00623.

${ }^{2}$ To whom correspondence should be addressed at Box 117, Children's Hospital Medical Center, 300 Longwood Avenue, Boston, MA 02115 .
}

by shaking the cage or by dropping the animal from a height of about $10 \mathrm{~cm}$. Electromyography revealed striking and constant electrical myotonia in all skeletal muscles tested. We now report the main genetic, behavioral, and histological properties of this inherited muscle disease (myotonia, mto) together with its electromyographic (EMG) features.

\section{Materials and Methods}

Animals. Since the coisogenic SWR/J strain is a relatively poor breeding strain, the present studies were carried out with affected mice descended from outcrosses. Homozygous $(m t o / m t o)$ and unaffected $(+/ ?)$ littermates were produced from matings of $m t o /+$ carriers. Affected mice were identified by 2 weeks of age when they showed prolonged "stiff" extensor postures of the limbs when gently dropped from a height of about $10 \mathrm{~cm}$. Heterozygous $(m t o /+)$ mice were identified by progeny testing. Control animals were age- and sex-matched C57BL/6J mice. All animals were housed in the Animal Care Facility of the Children's Hospital Medical Center.

EMG activity in mto homozygotes, heterozygotes, and controls. In order to study EMG activity, unanesthetized animals were restrained with tape and a ground wire was placed subcutaneously over the abdomen. Animals also were studied after anesthesia with tribromoethanol (approximately $0.3 \mathrm{ml}$, i.p.). A concentric needle electrode was inserted into the test muscle, including proximal and 
distal muscle groups of the forelimb and hindlimb, abdominal muscles, diaphragm, and tongue. All recordings were made with a Teca TE-4 electromyograph. Frequency response of EMG amplifiers was set at 300 to $3200 \mathrm{~Hz}$ ( $3 \mathrm{~dB}$ points). EMG output was connected to an audio speaker as well as the oscilloscope, and data were recorded on tape for later review.

Sciatic nerve stimulation. To study the effect of a controlled electrical nerve stimulus on EMG activity, we stimulated the sciatic nerve and recorded from the calf muscles (triceps surae) in anesthetized mto homozygotes and C57BL/6J controls. The sciatic nerve was exposed surgically in the thigh at the level of the hamstring muscles. The nerve was draped carefully over two 30 gauge silver wire electrodes connected to the stimulator. EMG activity was monitored simultaneously. An electrical stimulus of $0.05 \mathrm{msec}$ duration was applied over a frequency range of 0.5 to $100 \mathrm{~Hz}$.

Effect of peripheral nerve section and neuromuscular block. To determine whether the abnormal EMG activity in mto homozygotes was intrinsic to muscle, an EMG study of the calf muscles (triceps surae) was carried out in tribromoethanol-anesthetized mice before and after section of the sciatic nerve in the thigh and before and after subcutaneous administration of pancuronium bromide, $0.05 \mathrm{mg}$, to effect neuromuscular blockade.

Anatomical and histological studies. Mice were anesthetized with tribromoethanol $(0.025 \mathrm{ml} / \mathrm{gm})$ intraperitoneally and perfused through the heart with $1 \%$ paraformaldehyde and $1 \%$ glutaraldehyde to clear the vasculature, followed by $6 \%$ glutaraldehyde for 20 to $30 \mathrm{~min}$ (all in $100 \mathrm{~mm}$ sodium phosphate buffer, $\mathrm{pH} 7.4$, at $20^{\circ} \mathrm{C}$ ). 'Tissues were dissected immediately and consisted of spinal cord (at cervical, thoracic, and lumbar levels with associated ganglia), sciatic and posterior tibial nerves, and skeletal muscles of proximal and distal hindlimbs and forelimbs and of the tongue, all in predominantly transverse section. Tissues were washed in buffer, fixed in $2 \%$ osmium tetroxide and $1.5 \%$ potassium ferrocyanide, washed with sodium maleate buffer, and stained en bloc with $0.05 \%$ uranyl acetate, followed by dehydration with graded ethanols and infiltration with propylene oxide into plastic Dow epoxy resin 736 and nadic methyl anhydride (Polysciences, Warrington, PA). Blocks were hardened for $24 \mathrm{hr}$ at $70^{\circ} \mathrm{C}$ and sectioned at $1 \mu \mathrm{m}$ with a Sorvall MT2-B microtome, and sections were stained with toluidine blue $(0.5 \%$ toluidine blue and $0.5 \%$ sodium borate in $35 \%$ ethanol).

\section{Results}

Genetics. In order to locate the chromosomal position of $m t o$, several linkage crosses were made. Although we did not find its genetic location, we did eliminate close linkage to the following genes (given by chromosome): Chr 1 Idh-1 (isocitrate dehydrogenase-1, Chr $2 \mathrm{Cs}-1$ (catalase), Chr 3 Car-1 (carbonic anhydrase-1) and Amy1 (amylase-1), Chr 4 Gpd-1 (hexose 6-phosphate dehydrogenase), Chr $7 c$ (albino) and $H b b$ (hemoglobin $\beta$ chain), Chr 9 Mpi-1 (mannose phosphate isomerase-1), Chr 14 Np-1 (nucleoside phosphorylase-1), Chr 15 Gdc-1 (nicotinamide-adenine dinucleotide $\alpha$-glycerol phosphate dehydrogenase-1) and Gpt-1 (glutamic pyruvate transaminase-1), Chr 17 Glo-1 (glyoxylase-1), and Chr 19 Got1 (glutamate oxaloacetate transaminase-1).

The behavioral syndrome. At birth, homozygous mto mice produced by the mating of two $m t o /+$ carriers are behaviorally indistinguishable from their unaffected littermates. At 2 weeks of age, clinical myotonia is apparent. By 30 days of age, affected animals weigh about $10 \%$ less than their unaffected littermates, and this discrepancy increases to about $40 \%$ in adulthood.

Myotonia can be recognized in all mto homozygotes by a prolonged "stiff" extensor posture of the hindlimbs provoked by shaking the cage or by dropping the mouse from a height of about $10 \mathrm{~cm}$ onto its feet (Fig. 1). Repeated testing usually lessens the abnormality, but the full defect is again apparent after about $1 \mathrm{~min}$ of rest. If the mice are tested immediately after $2 \mathrm{hr}$ in a cold environment $\left(5^{\circ} \mathrm{C}\right)$, the extensor posture is elicited more easily and its duration is increased by about $50 \%$. The same treatment produces no apparent abnormality in littermates or known heterozygotes. When observed undisturbed in the cage, affected animals walk almost normally, but on close observation, the gait is slightly "stiff" and walking is slower than in unaffected littermates. Occasionally, an affected animal will begin to scratch with a hindlimb, but the usual rapid scratching movements do not occur. Homozygotes are capable of swim-
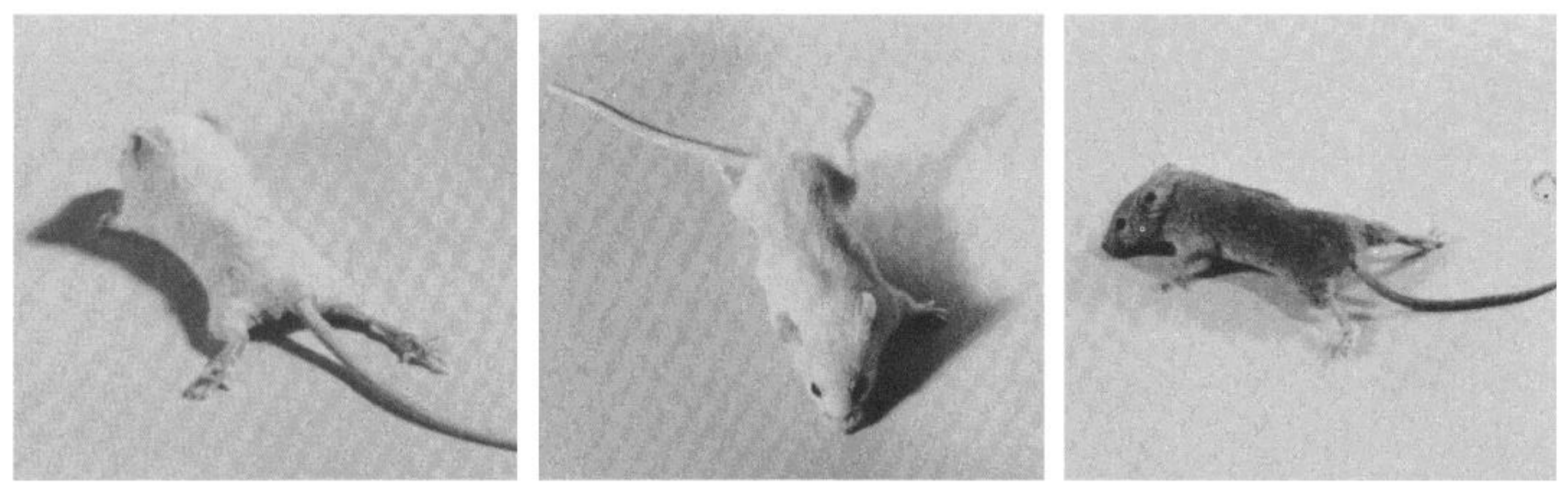

Figure 1. Behavioral myotonia in mto homozygotes age 60 days. The mice were dropped from a height of about $10 \mathrm{~cm}$. Note the abnormal extensor postures of the hindlimbs. 
ming, but alternate movements of the hindlimbs are not well coordinated. When tested in an ice water bath, affected mice exhibit a stiff extensor posturing of the hindlimbs which is most prominent during the 1st min and gradually subsides. After several minutes, affected mice reduce their activity and appear distressed sooner than unaffected littermates. Percussion of muscles in mto homozygotes (e.g., calf or anterior tibial muscle groups) occasionally produces a sustained local contraction with sustained posturing (e.g., flexion or dorsiflexion of the foot at the ankle).

Affected mice of an outcrossed (mixed) genetic background may live for a year or more and usually develop a mild dorsal kyphosis. The myotonia persists unchanged throughout adulthood. Weakness is not a prominent feature. Affected animals of both sexes may be fertile but are unreliable breeders. Throughout their lifetime, heterozygous $(m t o / t)$ mice are behaviorally indistinguishable from presumed $(+/+)$ littermates or $\mathrm{C} 57 \mathrm{BL} / 6 \mathrm{~J}$ control mice.

EMG activity in mto homozygotes, heterozygotes, and controls. With insertion of the needle electrode, myotonic discharges (high frequency repetitive discharges with recurrent variation of frequency and amplitude) were recorded from all muscles tested in the mto/mto homozygotes (Fig. 2). These discharges were easily elicited in all limb muscles, abdominal muscles, diaphragm, and tongue and were not affected appreciably by tribromoethanol anesthesia. When the needle was held in situ, myotonic discharges were often coincident with spontaneous "voluntary" movement in unanesthetized animals. In anesthetized animals, discharges could be evoked by percussion or stretching of the test muscle. The amplitude of the myotonic bursts varied from 400 to $1500 \mu \mathrm{V}$, while the frequency of the bursts varied from 60 to 160 $\mathrm{Hz}$ and, in some instances, reached a frequency of 500 $\mathrm{Hz}$. Episodes of myotonia could be easily identified by the characteristic "dive bomber" sound from the speaker and sometimes persisted for several seconds. These findings were observed in the more than 20 homozygotes tested and were independent of sex or age ( 19 days to 10 months). The age- and sex-matched control animals sometimes showed a brief volley of repetitive discharges with needle insertion (normal insertional activity) which always lasted for less than $200 \mathrm{msec}$ in contrast to the repetitive discharges in $m$ to animals which persisted for several seconds (Fig. 3). In the control animals, repetitive activity could not be elicited by percussion or stretching of the test muscle with the recording electrode in situ. Three proved heterozygotes (age, 130 to 160 days) could not be distinguished from age- and sex-matched C57RI /6.J controls studied under identical conditions.

Sciatic nerve stimulation. In mto homozygotes, electrical stimulation of the sciatic nerve evoked repetitive EMG activity in the calf muscle. This "myotonic" response was dependent on both the frequency of electrical stimulation and the number of stimuli applied in succession (train length). Thus, only 2 stimuli might be required to produce a repetitive burst when separated by a 10 msec interval (frequency, $100 \mathrm{~Hz}$ ), while a train of 8 might be required at $10 \mathrm{~Hz}$.

This "myotonic" response diminished with repeated trials at $10-\mathrm{sec}$ intervals but returned to base line after a 1 -min rest period. In control animals, no repetitive activity could be elicited with sciatic nerve stimulation under identical conditions at frequencies of 20 to $200 \mathrm{~Hz}$ and train lengths of 4 to 20 stimuli (Fig. 4).

Effect of peripheral nerve section and neuromuscular block. In anesthetized mto homozygotes, complete section of the sciatic nerve at the level of the hamstrings produced no change in the character of the electrical myotonia recorded from the calf muscle. Myotonia provoked by needle insertion or by stretching or percussion of the test muscle with the needle in situ persisted. Pancuronium bromide, $0.05 \mathrm{mg}$, abolished all EMG responses in calf muscle following a maximal electrical stimulus of the sciatic nerve within $2 \mathrm{~min}$. Under these conditions (and with spontaneous respirations abolished), there was no change in the character of the myotonia (Fig. 5). Additional confirmation that myotonia was not dependent on impulses from peripheral nerve was obtained with the demonstration that myotonia persisted in the amputated limb from an anesthetized mto homozygote for several minutes after amputation.

Anatomical and histological studies. By 70 days of age, all mto homozygotes showed increased muscle bulk in the neck and shoulder girdle muscles in comparison with age- and weight-matched controls as well as a pectus excavatum deformity. At 80 days, all mto homozygotes demonstrated upper thoracic kyphosis at the approximate T3-T5 level (Fig. 6). A translucent membrane covering the superior part of the eye was apparent in most animals after day 80 . No cataracts were observed.

A qualitative histopathological survey of several skeletal muscles showed no signs of muscle fiber necrosis, grouped atrophy, or inflammatory infiltrates. Central nucleation was equally frequent in mutant and control muscles. Only in older affected mice (225 days) were lesions seen by light microscopy, and these consisted of degeneration of myelinated axons in the ventrolateral funiculus at all levels of the spinal cord; however, nerve roots and mixed peripheral nerves did not appear to be affected qualitatively (Fig. 7).

\section{Discussion}

Myotonia is defined clinically as a delay in relaxation of a muscle or part of a muscle after voluntary forceful contraction or mechanical percussion (Munsat and Scheife, 1979) and is characterized electrophysiologically by high frequency repetitive discharges which show a recurrent variation of the frequency and amplitude (Goodgold and Eberstein, 1978). We have demonstrated that both the clinical and electromyographic features of myotonia are expressed in this single locus autosomal recessive disorder in mice. The myotonic signs of mto homozygotes are exacerbated by cold and ameliorated by repeated activity. A similar "warm up" phenomenon also can be demonstrated with direct peripheral nerve stimulation of affected muscle. Repetitive discharges can be elicited by electrical stimulation of the peripheral nerve. This abnormal response fatigues with repeated trials but recovers with rest. The myotonic discharges of the mto mutant are not altered by peripheral nerve section or by the neuromuscular blocking agent pancuronium bromide, 


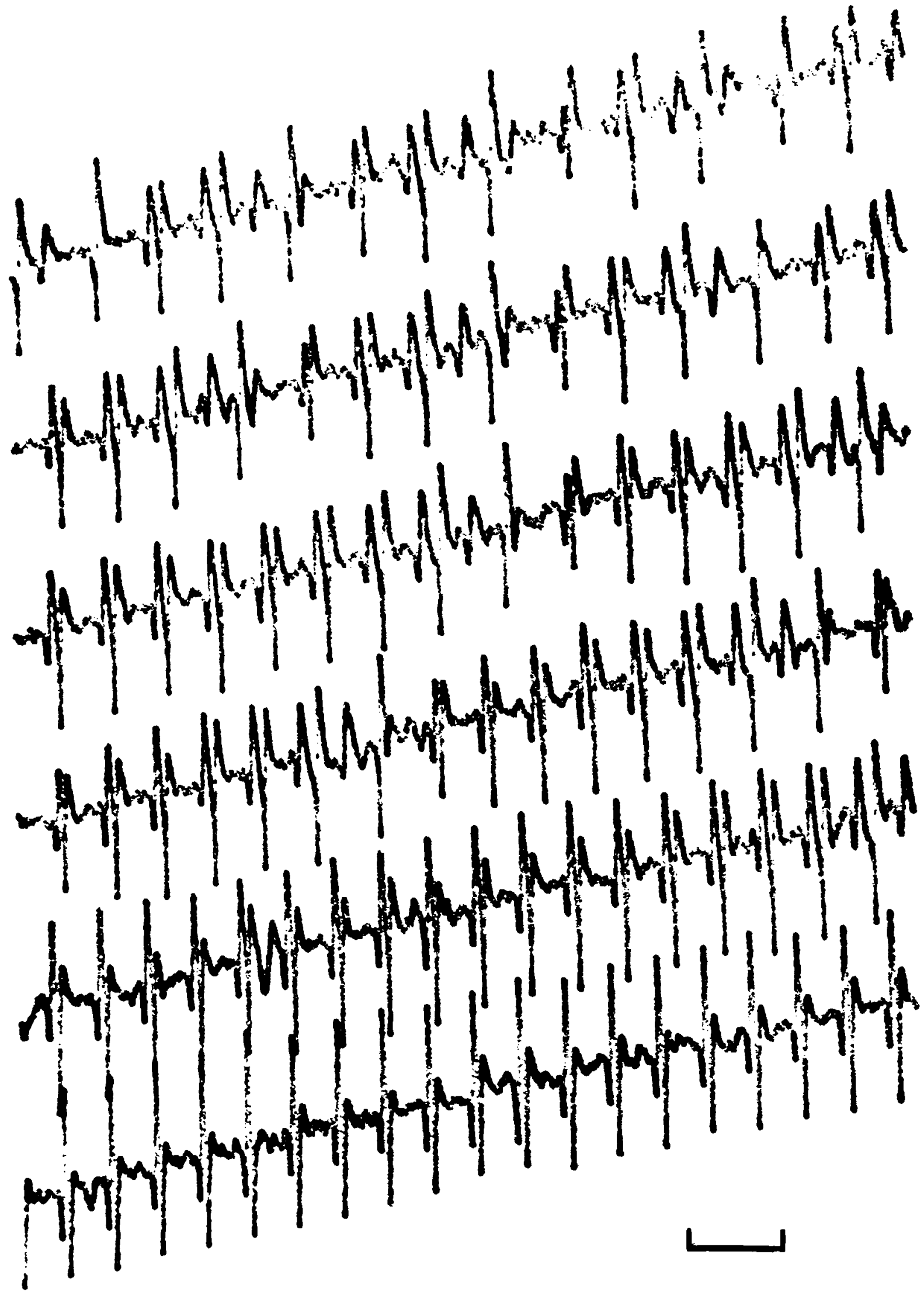

Figure 2. Spontaneous myotonic discharge recorded from the hamstring muscle in an unanesthetized mto homozygote. The tracings represent a continuous record from bottom to top. High frequency repetitive discharges with variation of frequency and amplitude are apparent. Calibration bar, $1 \mathrm{mV} ; 10 \mathrm{msec}$. 

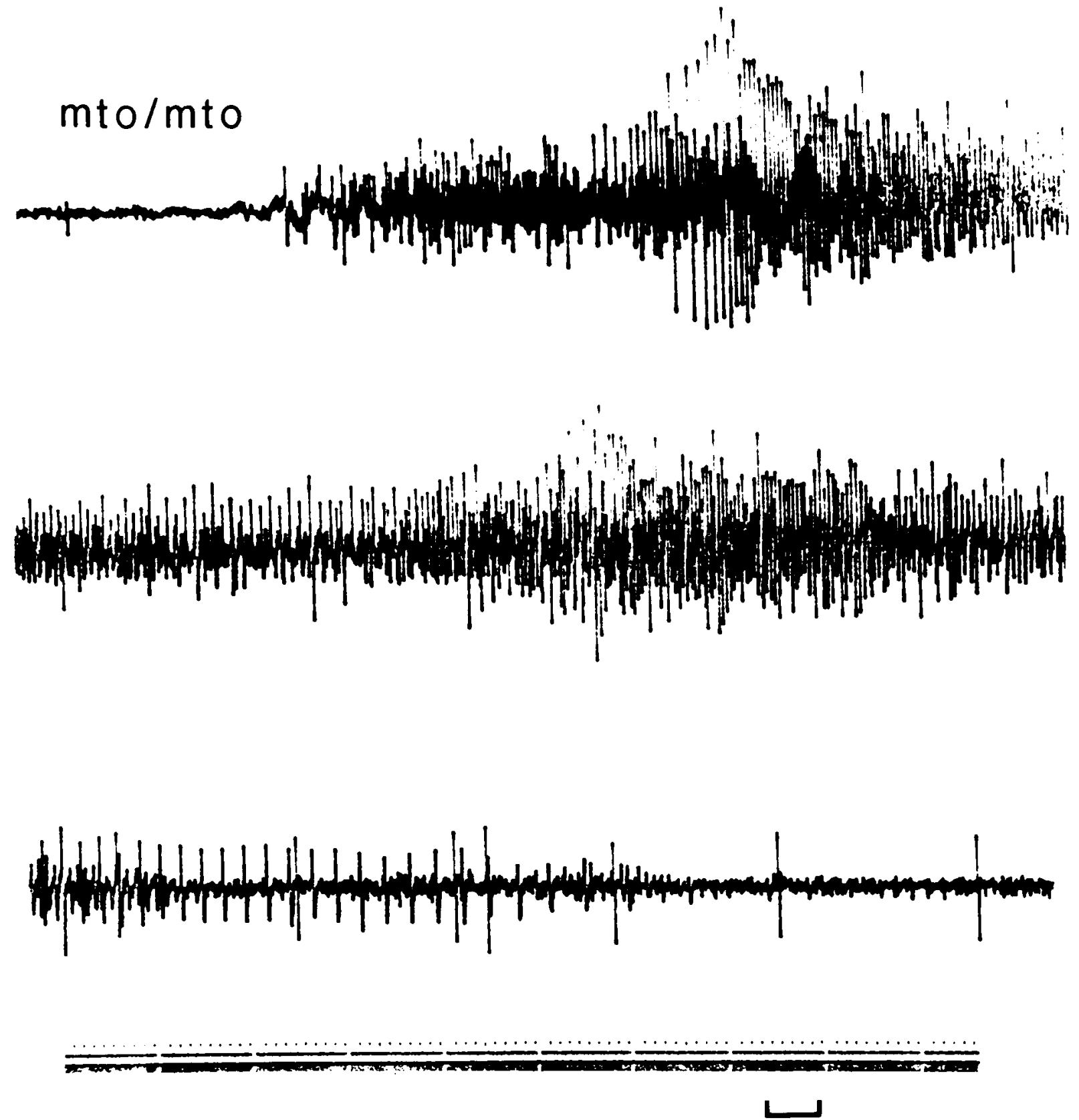

\section{control}
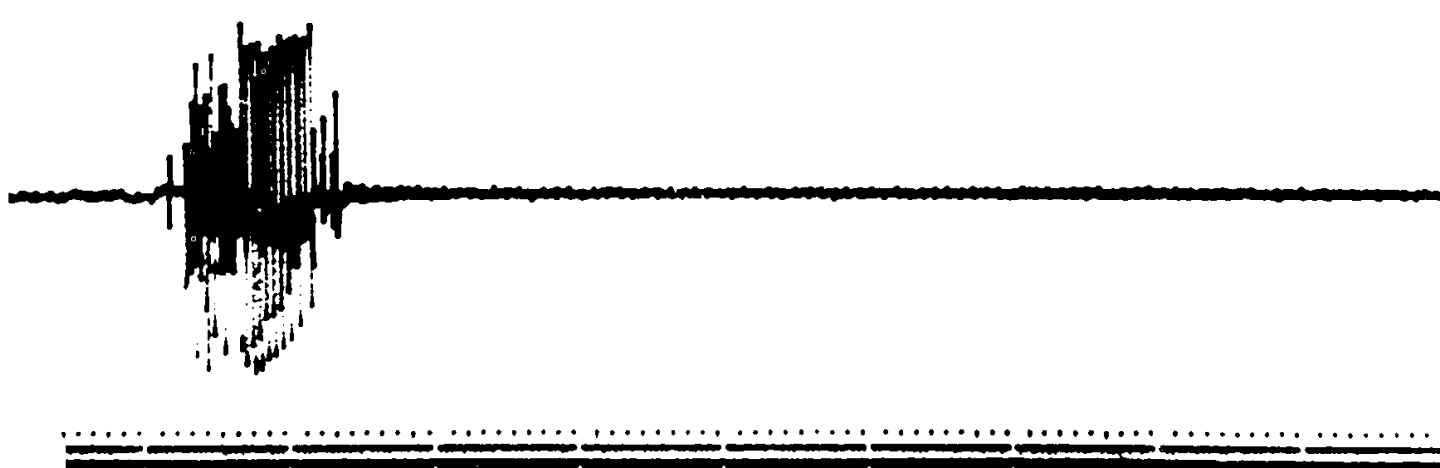

Figure 3. EMG activity following needle insertion recorded from the hamstring muscles of an anesthetized mto homozygote and a C57BL $/ 6 \mathrm{~J}+1+$ control animal. The upper three tracings represent a continuous record from top to bottom. High frequency repetitive discharges with variation in frequency and amplitude are apparent and persist for several seconds. Additional bursts of myotonia could be elicited by minimal movement of the needle in situ or by stretching or percussion of the test muscle. In the control animal, repetitive discharges occur with needle insertion (normal insertional activity), but these persist for no longer than $200 \mathrm{msec}$. With the needle in situ, no further repetitive activity could be elicited with percussion or stretching of the test muscle. Calibration bar, $1 \mathrm{mV}$; 50 msec. 

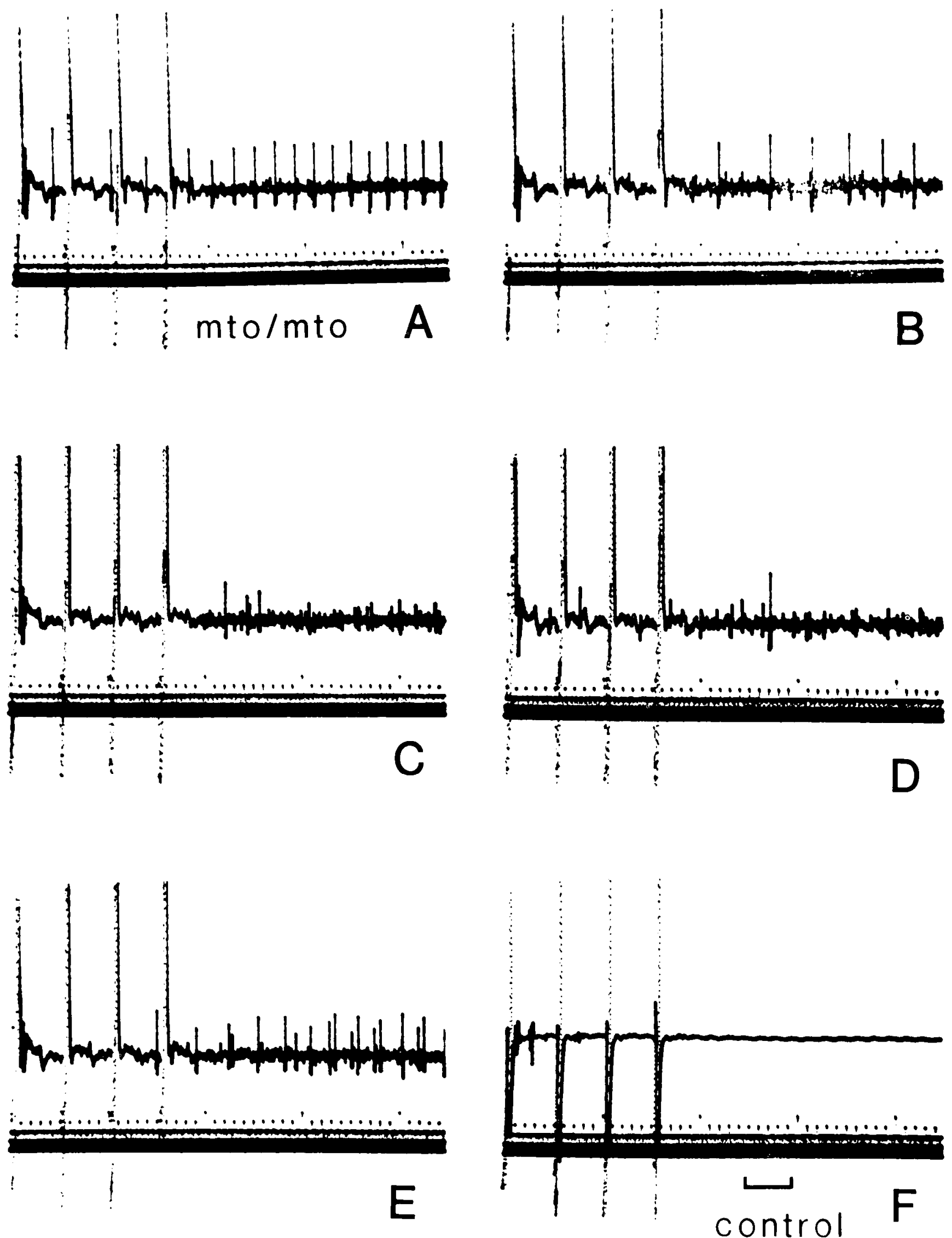

Figure 4. EMG activity recorded from the calf muscle of an mto homozygote and control animal following repetitive electrical stimulation of the sciatic nerve. In each case, the nerve was stimulated with a train of 4 stimuli $(0.05$ msec duration) at a frequency of $20 \mathrm{~Hz}$. Recordings $A$ to $D$ were obtained at consecutive 10 -sec intervals. Recording $E$ was obtained 1 min later. The repetitive response $(A$ and $E)$ persists for at least $250 \mathrm{msec}$ after the stimulus train is complete. Note the progressive decrease in the "myotonic" response $(A$ to $D)$ followed by the return to base line after the 1-min rest interval. Under identical conditions, no repetitive response could be elicited in the C57BL/6J control animal $(F)$. (In C57BL/6J control animals, no myotonic response could be elicited at stimulus frequencies as high as $200 \mathrm{~Hz}$ and/or train lengths up to 20 stimuli.) Calibration bar, $100 \mu \mathrm{V}$; 50 msec. 
A

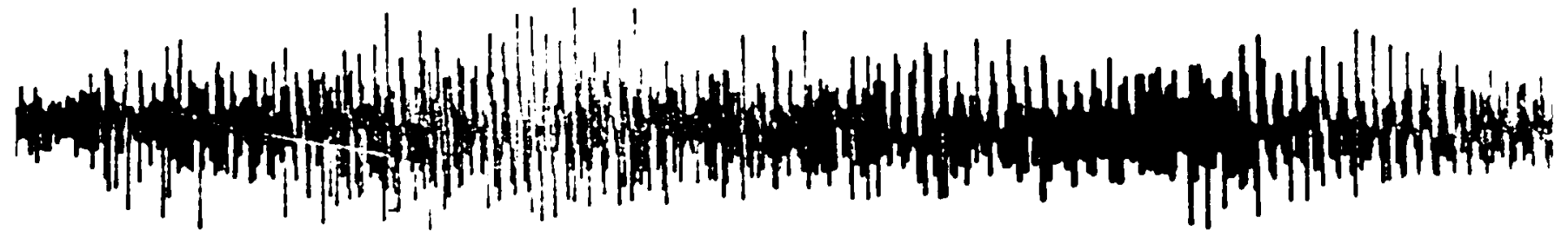

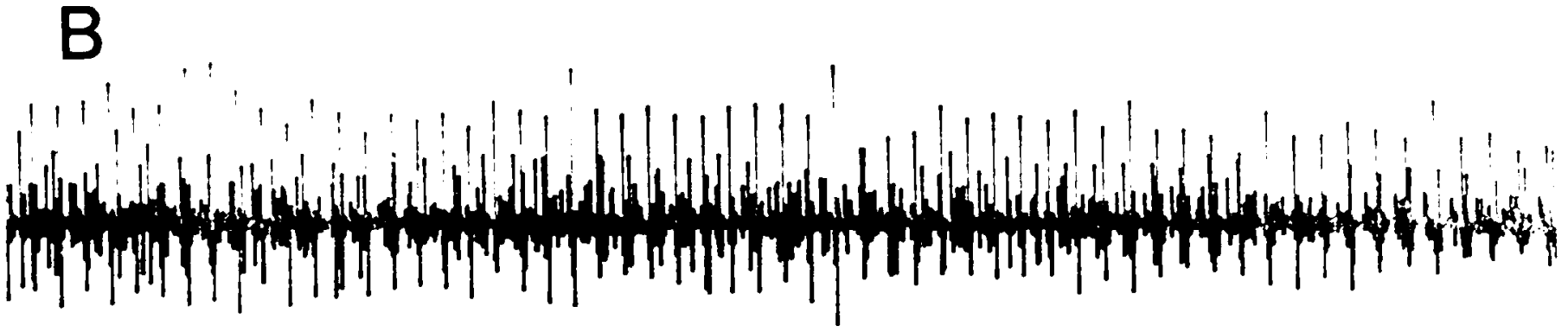
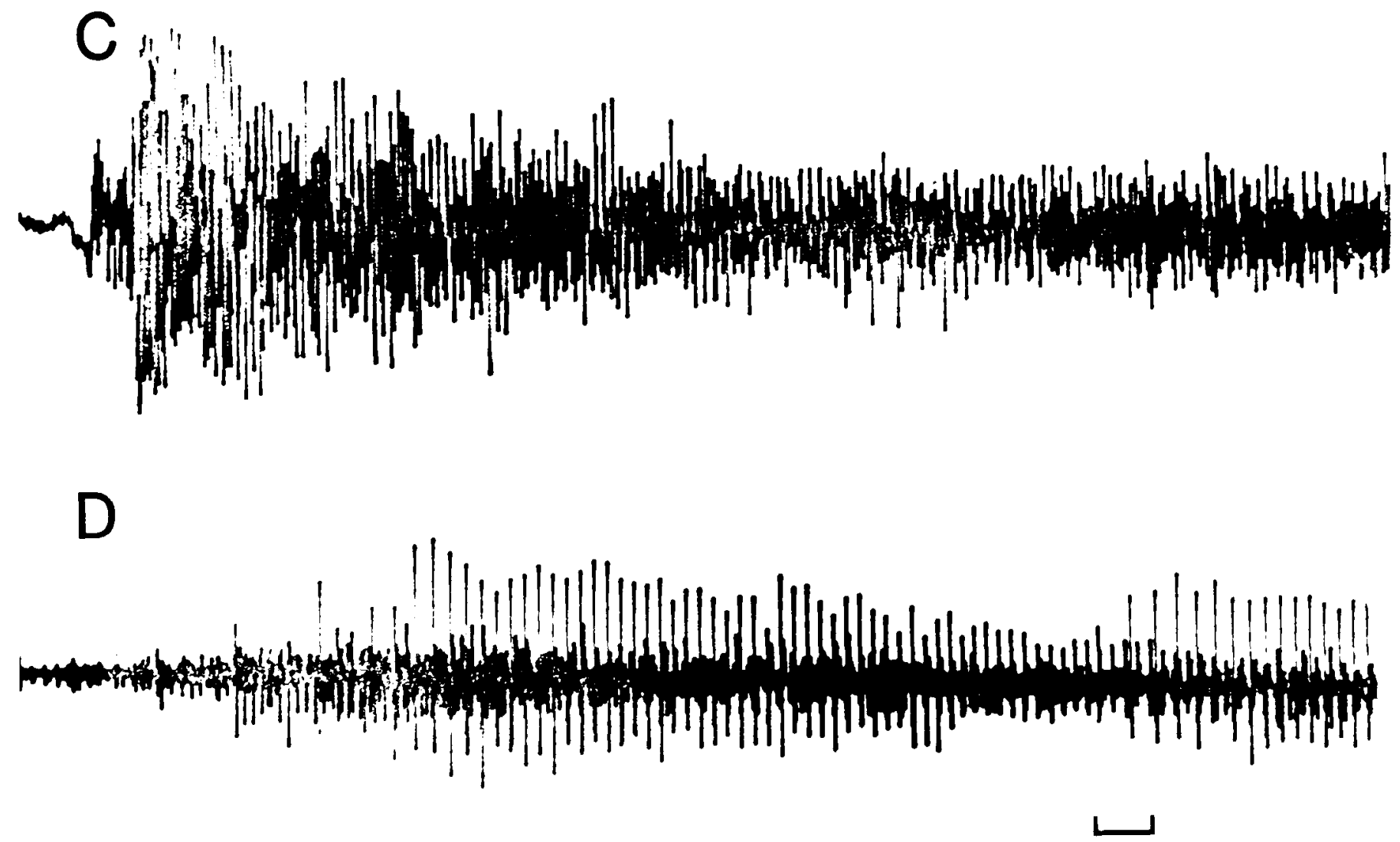

Figure 5. Effect of sciatic nerve section or pancuronium bromide on myotonia in mto homozygotes. EMG activity following needle insertion was recorded from the calf muscle $(A)$ before sciatic nerve section, $(B)$ immediately after sciatic nerve section (same animal as $A$ ), $(C)$ before pancuronium bromide, and $(D) 3 \mathrm{~min}$ after subcutaneous pancuronium bromide, $0.05 \mathrm{mg}$ (same animal as $C$ ). Myotonia persists after peripheral nerve section or neuromuscular block. The significance of the apparent change in discharge frequency after nerve section or neuromuscular block has not been determined. Calibration bar, $100 \mu \mathrm{V} ; 50 \mathrm{msec}$. 

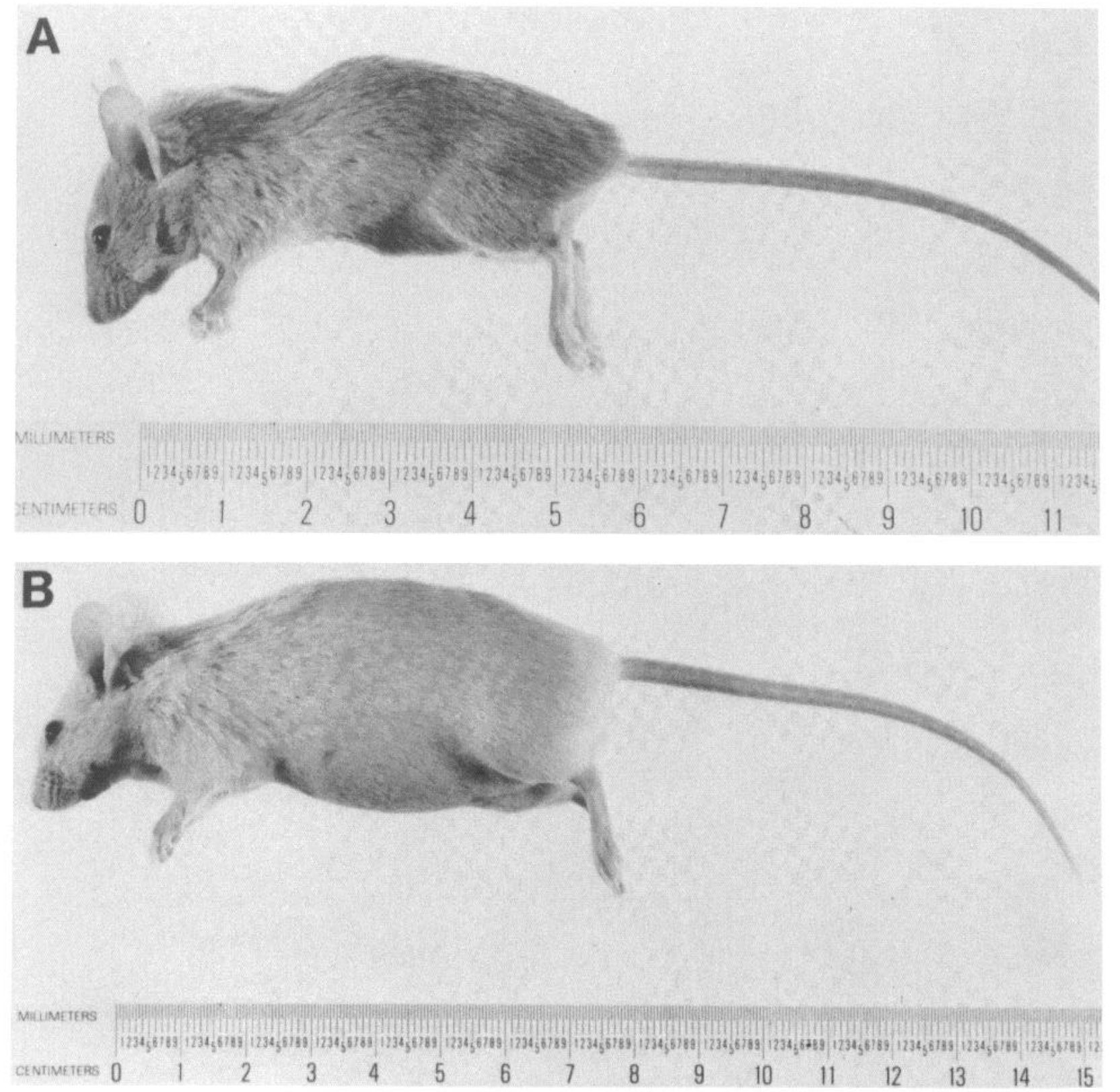

Figure 6. Thoracic kyphosis in an mto homozygote age 140 days $(A) . B$ shows an unaffected $+/$ ? littermate with a normal appearance. Mice were anesthetized with tribromoethanol, $0.025 \mathrm{ml} / \mathrm{gm}$.

suggesting that these discharges do not originate in the peripheral nerve. Thus, the mto mutant exhibits classical myotonia, presumably directly referable to muscle, as previously recognized in man (Denny-Brown and Nevin, 1941) and the myotonic goat (Brown and Harvey, 1939).

Thus far, qualitative histological studies at the light microscopic level have demonstrated degeneration of myelinated axons in the ventrolateral funiculus of the spinal cord in older animals. These changes do not appear to relate to the pathogenesis of the myotonia and are not apparent until months after the development of the behavioral and electrical myotonic disorder. Qualitative histological study revealed lesions neither in skeletal muscle nor in peripheral nerves.

It should be pointed out that the myotonia of the mto mutant exhibits important features which contrast with the "neural pseudo-myotonia" described in dystrophic (dy) mice (Silverman and Atwood, 1980). Homozygous mutants $d y$ and $d y^{2 J}$ exhibit myotonia-like behavior (Gilbert et al., 1973) and EMG features of myotonia (Pachter et al., 1974), but both the electrical features and delayed muscle relaxation are abolished by nerve section or by neuromuscular blockade (Eberstein et al., 1975). A neural origin for the pseudomyotonia has been proposed based on the observation that many spinal root axons of $d y$ and $d y^{2 J}$ mutants are not insulated by myelinated sheaths (Bradley and Jenkison, 1973) and that abnormal impulse propagation occurs between apposed fibers (Huizar et al., 1975; Rasminsky, 1978).

The mto mutant may serve as a conveniently accessible model for clarifying the mechanisms and therapy of 

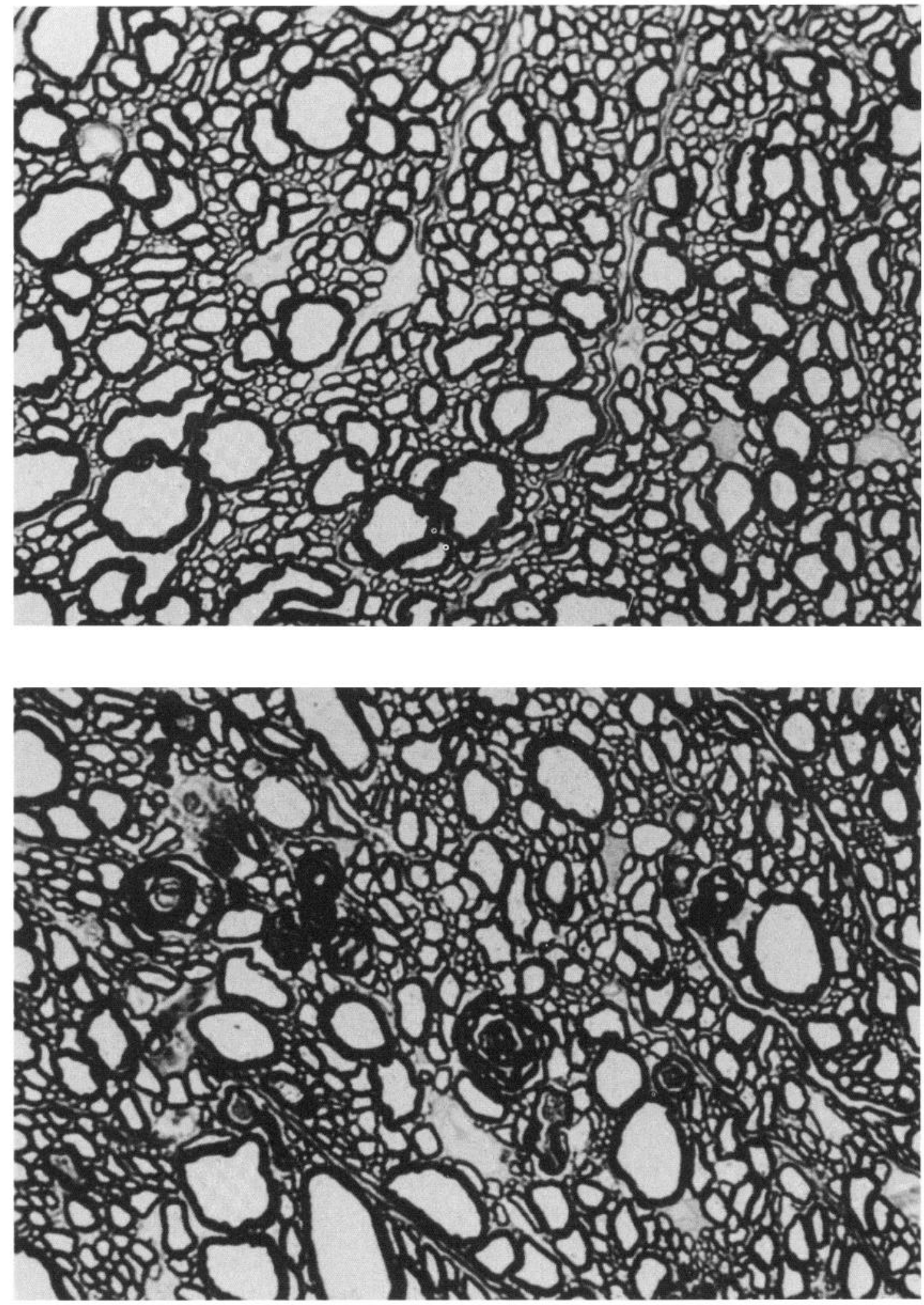

Figure 7. Ventrolateral white matter funiculus of lumbar spinal cord from 225-day-old mice. Plastic-embedded, 1- $\mu \mathrm{m}$ sections were stained with alkaline toluidine blue. The upper panel shows a field from a $+/+$ mouse at a magnification of $\times 60$ with intact myelinated fibers of various calibers. The lower panel shows a field from an $\mathrm{mto} / \mathrm{mto}$ mouse at the same magnification with numerous myelinated axons at various stages of degeneration. Lesions were minimal or absent in spinal cord at younger ages when the myotonia was already prominent.

myotonic diseases. Myotonic muscular dystrophy (myotonia dystrophica) and the common form of myotonia congenita are inherited as an autosomal dominant trait, while a less common form of myotonia congenita may be inherited in an autosomal recessive manner. The myotonic goat, despite the uncertainty about the mode of inheritance and the inconvenience of its use as a laboratory animal, has been of great value in the development 
of the in vitro biopsy study method, localization of the functional myotonic lesion to the skeletal muscle membrane, and elucidation of a specific block in chloride conductance (Bryant, 1979). The mto/mto mouse would seem to offer significant advantages for analysis by the new methods of cellular and developmental biology and molecular genetics.

\section{References}

Bradley, W. G., and M. Jenkison (1973) Abnormalities of peripheral nerves in murine dystrophy. J. Neurol. Sci. 317: 132142.

Brown, G. L., and A. M. Harvey (1939) Congenital myotonia in the goat. Brain 62: 341-363.

Bryant, S. H. (1979) Myotonia in the goat. Ann. N. Y. Acad. Sci. 317: 314-324.

Denny-Brown, D., and S. Nevin (1941) The phenomenon of myotonia. Brain 64: 1-18.

Eberstein, A., J. Goodgold, and B. R. Pachter (1975) Effect of curare on electromyographic and contractile responses in the myotonic mouse. Exp. Neurol. 49: 612-616.
Gilbert, J. J., M. C. Steinberg, and B. Q. Banker (1973) Ultrastructural alterations of the motor endplate in myotonic dystrophy of the mouse $\left(d y^{2 J} / d y^{2 J}\right)$. J. Neuropathol. Exp. Neurol. 32: 345-364.

Goodgold, J., and A. Eberstein (1978) Electrodiagnosis of Neuromuscular Diseases, Ed. 2, p. 280, Williams and Wilkins Co., Baltimore.

Huizar, P., M. Kuno, and Y. Miyata (1975) Electrophysiological properties of spinal motoneurones of normal and dystrophic mice. J. Physiol. (Lond.) 248: 231-246.

Munsat, T. L., and R. T. Scheife (1979) Myotonia. In Clinical Neuropharmacology, H. L. Klawans, ed., Vol. 4, pp. 83-107, Raven Press, New York.

Pachter, B. R, J. Davidowitz, A. Eberstein, and G. M. Breinin (1974) Myotonic muscle in mouse: A light and electron microscopic study in serial sections. Exp. Neurol. 45: 462-473.

Rasminsky, M. (1978) Ectopic generation of impulses and crosstalk in spinal nerve roots of "dystrophic" mice. Ann. Neurol. 3: $351-357$.

Silverman, H., and H. L. Atwood (1980) Increase in oxidative capacity of muscle fibers in dystrophic mice and correlation with overactivity in these fibers. Exp. Neurol. 68: 97-113. 